\title{
Plant-based biosensors for detecting CRISPR-mediated genome engineering
}

Guoliang Yuan ${ }^{1,2}$, Md. Mahmudul Hassan ${ }^{1,2,3}$, Tao Yao ${ }^{1,2}$, Haiwei Lu ${ }^{1, \dagger}$, Michael Melesse Vergara ${ }^{1}$, Jesse L. Labbé ${ }^{1}$, Wellington Muchero ${ }^{1,2}$, Changtian Pan ${ }^{4}$, Jin-Gui Chen ${ }^{1,2}$, Gerald A. Tuskan ${ }^{1,2}$, Yiping Qi ${ }^{4,5}$, Paul E. Abraham ${ }^{1 *}$, Xiaohan Yang ${ }^{1,2} *$

${ }^{1}$ Biosciences Division, Oak Ridge National Laboratory, Oak Ridge, TN 37831, USA

${ }^{2}$ The Center for Bioenergy Innovation, Oak Ridge National Laboratory, Oak Ridge, TN 37831, USA

${ }^{3}$ Department of Genetics and Plant Breeding, Patuakhali Science and Technology University, Dumki, Patuakhali-8602, Bangladesh

${ }^{4}$ Department of Plant Science and Landscape Architecture, University of Maryland, College Park, MD 20742, USA

${ }^{5}$ Institute for Bioscience and Biotechnology Research, University of Maryland, Rockville, MD 20850, USA

*Corresponding authors: Paul Abraham (abrahampe@ornl.gov); Xiaohan Yang (yangx@ornl.gov)

$\dagger$ Current address: Department of Academic Education, Central Community College - Hastings, Hastings, NE 68902, USA

\section{Running title: Biosensors for detecting CRISPR systems}

\begin{abstract}
CRISPR/Cas has recently emerged as the most reliable system for genome engineering in various species. However, concerns about risks associated with CRISPR/Cas9 technology are increasing on potential unintended DNA changes that might accidentally arise from CRISPR gene editing. Developing a system that can detect and report the presence of active CRIPSR/Cas tools in biological systems is therefore very necessary. Here, we developed the real-time detection systems that can spontaneously indicate CRISPR-Cas tools for genome editing and gene regulation including CRISPR/Cas9 nuclease, base editing, prime editing and CRISPRa in plants. Using the fluorescence-based molecular biosensors, we demonstrated that the activities of CRISPR/Cas9 nuclease, base editing, prime editing and CRIPSRa can be effectively detected in transient expression via protoplast transformation and leaf infiltration (in Arabidopsis, poplar, and tobacco) and stable transformation in Arabidopsis.
\end{abstract}

Keywords: CRISPR, genome editing, biosensor, detection, transient gene expression. 


\section{Introduction}

Different CRISPR/Cas-based genome engineering tools, such as base editors (BEs), prime editors (PEs), CRISPR activation (CRISPRa) and interference, have been developed in life sciences. ${ }^{1,2}$ Although CRISPR/Cas is a revolutionary technology, it poses potential biosecurity risks, increasing ethical and safety concerns. ${ }^{3,4}$ Therefore, it is necessary to develop a system that can detect the presence of active CRISPR/Cas tools in biological systems. Multiple CRISPR/Cas technologies have been tested in multiple plant species. ${ }^{5}$ However, methods for detecting CRISPR systems have not yet been reported in plants. Here, we developed fluorescence-based biosensors to detect in planta activities of CRISPR/Cas9, BEs, PEs, and CRISPRa.

\section{Results and Discussion}

To detect active CRISPR/Cas9 nucleases, we created two variants of biosensor 1 (BS1), BS1-1 and BS1-2, which contain different frameshift GFP mutants ( $g f p l a$ or $g f p l b$ ), and single guide RNAs (sgRNAs) targeting $g f p l a$ and $g f p 1 b$, respectively (Figure 1a). T-DNA vector p201N-Cas9 6 was co-expressed with BS1-1/BS1-2 to detect CRISPR/Cas9-mediated gene editing, which generates functional GFP through insertion/deletions in gfpla or gfplb, in Arabidopsis thaliana protoplasts and Nicotiana benthamiana (tobacco) leaves. Bright green fluorescence was observed in both the protoplasts transformed with 35Sp:GFP (positive control) and those co-transfected with p201N-Cas9 and BS1-1/BS1-2, but not in the protoplasts transformed with BS1-1/BS1-2 only (negative control) (Figure 1b). Furthermore, $\sim 34 \%$ and $\sim 15 \%$ of the cells exhibited GFP signals in the positive control and the samples with Cas9-BS1 co-transformation, respectively (Figure 1c). Similar results were obtained through Agrobacterium-mediated leaf infiltration in tobacco (Figure 1b). These results indicate that BS1 can be used as an efficient biosensor for detecting active Cas9 in plant systems.

To detect active adenine base editors (ABEs) and PEs, we generated BS2 and BS3 by integrating a dark-GFP mutant ( $g f p 2$ ) harboring a premature termination codon (PTC) with corresponding sgRNA and pegRNA (Figure $1 \mathrm{~d}$ and e). As such, an active ABE or PE will ${ }^{7,8}$ and BS3 to generate a functional GFP protein. Two BS2 variants, BS2-1 ( $g f p 2 a, \mathrm{Q} 69>\mathrm{TAG})$ and BS2-2 ( $g f p 2 b$, Q80>TAG), were tested by co-expressing with a plant adenine base editor PABE-7 ${ }^{9}$ using protoplast transformation in Arabidopsis, poplar '717' (Populus tremula x alba INRA '717-1B4'), poplar 'WV94' (Populus deltoides 'WV94'), and tobacco leaf infiltration. In Arabidopsis, '7171B4' and 'WV94', strong GFP fluorescence was observed in both the positive control (35Sp:GFP) and the protoplasts co-transfected with PABE-7 and BS2-1/BS2-2 but not those transformed with BS2-1/BS2-2 alone (Figure 1f), indicating that base editing mediated by PABE-7 successfully rescued the gfp 2 mutants. In Arabidopsis, $\sim 64 \%$ and $\sim 45 \%$ of the cells exhibited GFP fluorescence in the positive control and the samples with BS2-PABE-7 co-transformation, respectively (Figure 1g). In poplar ' $717-1 \mathrm{~B} 4$ ', GFP fluorescence was detected in $\sim 71 \%, \sim 45 \%$ and $35 \%$ of the cells in the positive control, the samples co-transfected with PABE-7 and BS2-1, and the samples cotransfected with PABE-7 and BS2-2, respectively (Figure 1h). In poplar 'WV94', GFP fluorescence was detected in $\sim 73 \%$ and $38 \%$ of the cells in the positive control and the protoplasts co-transformed with PABE-7 and BS2-1/BS2-2, respectively (Figure 1i). These results indicate that both BS2-1 and BS2-2 are relatively efficient for detecting base editing in protoplasts derived 
from herbaceous and woody plants. Similar results were also observed in the tobacco leaf (Figure 1f). Furthermore, after co-transformation of the BS2-1 and PABE-7 using floral dip, GFP signals were observed in the primary root of Arabidopsis transgenic lines (Figure 1j). Taken together, results from both transient gene expression and stable transformation demonstrate that BS2 is a reliable and robust system for the detection of ABE-mediated gene editing in plants.

BS3 containing the $g f p 2 b$ mutant and a pegRNA was tested by co-expressing with a plant prime editor PPE2 ${ }^{10}$ in Arabidopsis protoplasts. GFP signals were detected in the protoplasts with the positive control (35Sp:GFP) and those co-transformed with BS3 and PPE2, but not in the protoplasts transformed with BS3 alone (Figure 1k). This result indicates that the prime editing successfully rescued the $g f p 2 b$ mutant, producing functional GFP proteins. Notably, a relatively low percentage (5\%) of protoplasts displayed GFP signals in the co-transformation of BS3 and PPE2 in comparison with that $(63 \%)$ in the positive control (Figure 11). This could be explained by the lower efficiencies of the current generation of PEs in plants. ${ }^{2}$ Therefore, while BS3 can be used to detect active prime editing through protoplast transient expression, the efficiency needs to be improved in the future.

To detect active CRISPRa, we generated BS4 by incorporating the ProOsTPR-like:mCherry and a sgRNA targeting ProOsTPR-like ${ }^{11}$ (Figure $1 \mathrm{~m}$ ). As such, mCherry signals are expected to be much stronger in the presence of a CRISPRa system. The efficacy of BS4 was tested by co-expressing BS4 and a plant CRISPRa system, CRISPR-Act3.0 ${ }^{11}$ in Arabidopsis protoplasts. Strong red fluorescence was observed in $75 \%$ of protoplasts in the positive control (35Sp:mCherry) and $32 \%$ of those in the samples co-transformed with BS4 and CRISPR-Act 3.0 whereas no red fluorescence signal was detected in the protoplasts transformed with BS4 alone (Figure 1n and o). This result indicates that BS4 is a highly efficient biosensor for detecting CRISPRa activity in plants.

In summary, we developed efficient biosensors for detecting four different CRISPR tools, providing new opportunities to monitor and evaluate the efficiency of different CRISPR/Cas-based genome engineering tools in real-time and may serve as an early-detection system for unwanted genome engineering.

\section{Materials and methods}

\section{Construction of vector}

To build the BS1, the mutant $g f p l a$ and $g f p l b$ were created by inserting a gblcoks containing frame shift mutation sequences at the 5' end of GFP coding sequence of pGFPGUSPlus (Plasmid \#64401 in addgene) using the NEBuilder HiFi DNA Assembly Cloning Kit (New England BioLabs, Catalog \#E5520S). Then, a gblocks harboring a U6 promoter, sgRNA and terminator was assembled into the mutant $g f p l a$ and $g f p l b$ through NEBuilder HiFi DNA Assembly. To build the BS2 and BS3, the mutant $g f p 2 a$ and $g f p 2 b$ were created by replacing the GFP coding sequence of pGFPGUSPlus with a gblcoks containing PTC mutation. BS2 was then assembled by inserting a gblocks harboring a U6 promoter, sgRNA and terminator into the mutant $g f p 2 a$ and $g f p 2 b$ through NEBuilder HiFi DNA Assembly. BS3 was assembled by inserting a gblocks harboring a U6 promoter, pegRNA and terminator into the mutant $g f p 2 b$. To build the BS4, a gblocks harboring a 
U3 promoter, sgRNA and terminator was inserted into the vector ProOsTPR-like:mCherry ${ }^{11}$ using NEBuilder HiFi DNA Assembly. All gblocks were synthesized from Integrated DNA Technologies. All plasmids were confirmed by Sanger sequencing.

\section{Protoplast transformation}

The isolation and transformation of Arabidopsis and poplar protoplast were performed as described previously. ${ }^{12}$

\section{Arabidopsis transformation}

The floral dip method of transformation was performed by immersion of Arabidopsis wild type 'Col-0' inflorescences in a suspension of Agrobacterium 'GV3101' with modification as described by Yuan et al. ${ }^{13}$

\section{Tobacco leaf infiltration}

$N$. benthamiana leaves were infiltrated using a syringe without a needle as described by Li. ${ }^{14}$

\section{Author contributions}

G.Y., P.A. and X.Y conceived the research. G.Y., T.Y. H.L. and M.H. conducted the experiments. G.Y. wrote the paper. M.H., H.L., T.Y., M.V., J. L., W.M., J.-G.C., G.T., P.A. and X.Y. revised the manuscript. C.P. and Y.Q. provided CRISPRa reagents and revised the manuscript.

\section{Acknowledgements}

This work was funded by the Genomic Science Program of the U.S. Department of Energy, Office of Science, Office of Biological and Environmental Research (BER) as part of the Secure Ecosystems Engineering and Design research program in the Secure Biosystems Design Scientific Focus Area (SFA). Oak Ridge National Laboratory is managed by UT-Battelle, LLC for the U.S. Department of Energy under Contract Number DE-AC05-00OR22725. This work was also supported by the National Science Foundation Plant Genome Research Program (award no. IOS1758745 and IOS-2029889).

\section{Competing interests}

The authors declare no conflict of interests.

\section{Reference}

(1) La Russa, M. F., and Qi, L. S. (2015) The new state of the art: Cas9 for gene activation and repression, Mol Cell Biol 35, 3800-3809.

(2) Hassan, M. M., Zhang, Y., Yuan, G., De, K., Chen, J.-G., Muchero, W., Tuskan, G. A., Qi, Y., and Yang, X. (2021) Construct design for CRISPR/Cas-based genome editing in plants, Trends Plant Sci, https://doi.org/10.1016/j.tplants.2021.1006.1015.

(3) Abraham, P. E., Labbé, J. L., and McBride, A. A. (2020) Advancing how we learn from biodesign to mitigate risks with next-generation genome engineering, BioDesign Research 2020, 9429650. 
(4) Brokowski, C., and Adli, M. (2019) CRISPR ethics: moral considerations for applications of a powerful Tool, J Mol Biol 431, 88-101.

(5) Hassan, M. M., Yuan, G., Chen, J.-G., Tuskan, G. A., and Yang, X. (2020) Prime editing technology and its prospects for future applications in plant biology research, BioDesign Research 2020, 9350905.

(6) Jacobs, T. B., LaFayette, P. R., Schmitz, R. J., and Parrott, W. A. (2015) Targeted genome modifications in soybean with CRISPR/Cas9, BMC Biotechnol 15, 16.

(7) Yuan, G., Hassan, M. M., Yao, T., Lu, H., Vergara, M. M., Labbé, J. L., Muchero, W., Pan, C., Chen, J.-G., Tuskan, G. A., Qi, Y., Abraham, P. E., and Yang, X. (2021) Plant-based biosensors for detecting CRISPR-mediated genome engineering ACS Synth Biol, Under Review

(8) Duan, L., Ouyang, K., Xu, X., Xu, L., Wen, C., Zhou, X., Qin, Z., Xu, Z., Sun, W., and Liang, Y. (2021) Nanoparticle Delivery of CRISPR/Cas9 for Genome Editing, Frontiers in Genetics 12.

(9) Li, C., Zong, Y., Wang, Y. P., Jin, S., Zhang, D. B., Song, Q. N., Zhang, R., and Gao, C. X. (2018) Expanded base editing in rice and wheat using a Cas9-adenosine deaminase fusion, Genome Biology 19.

(10) Lin, Q., Jin, S., Zong, Y., Yu, H., Zhu, Z., Liu, G., Kou, L., Wang, Y., Qiu, J. L., Li, J., and Gao, C. (2021) High-efficiency prime editing with optimized, paired pegRNAs in plants, Nat Biotechnol.

(11) Pan, C., Wu, X., Markel, K., Malzahn, A. A., Kundagrami, N., Sretenovic, S., Zhang, Y., Cheng, Y., Shih, P. M., and Qi, Y. (2021) CRISPR-Act3.0 for highly efficient multiplexed gene activation in plants, Nat Plants.

(12) Xie, M., Muchero, W., Bryan, A. C., Yee, K., Guo, H.-B., Zhang, J., Tschaplinski, T. J., Singan, V. R., Lindquist, E., Payyavula, R. S., Barros-Rios, J., Dixon, R., Engle, N., Sykes, R. W., Davis, M., Jawdy, S. S., Gunter, L. E., Thompson, O., DiFazio, S. P., Evans, L. M., Winkeler, K., Collins, C., Schmutz, J., Guo, H., Kalluri, U., Rodriguez, M., Feng, K., Chen, J.-G., and Tuskan, G. A. (2018) A 5-Enolpyruvylshikimate 3-Phosphate Synthase Functions as a Transcriptional Repressor in Populus The Plant Cell 30, 1645-1660.

(13) Yuan, G., Ahootapeh, B. H., Komaki, S., Schnittger, A., Lillo, C., De Storme, N., and Geelen, D. (2018) PROTEIN PHOSHATASE 2A B' $\alpha$ and $\beta$ Maintain Centromeric Sister Chromatid Cohesion during Meiosis in Arabidopsis, Plant Physiology 178, 317-328.

(14) Li, X. (2011) Infiltration of Nicotiana benthamiana Protocol for Transient Expression via Agrobacterium, Bio-protocol 1, e95.

Figure 1. Detection of CRISPR tools for genome editing and gene regulation in plants. (a) BS1 for detection of the CRISPR/Cas9 nuclease. (b) Detection of CRISPR/Cas9 by BS1 through Arabidopsis protoplast transformation and tobacco leaf infiltration. (c) Statistical analysis of GFP-positive cells with and without the CRISPR/Cas9 in Arabidopsis. (d) BS2 for detection of the adenine base editor (ABE). (e) BS3 for detection of the prime editor. (f) Detection of an ABE by BS2 through protoplast transformation in Arabidopsis and poplar, and tobacco leaf infiltration. (g-i) Statistical analysis of GFP-positive cells with and without ABE in Arabidopsis, poplar '717', and poplar 'WV94'. (j) Detection of an ABE by BS2 through stable transformation in Arabidopsis. 
(k) Detection of prime editor PPE2 by BS3 through Arabidopsis protoplast transformation. (1) Statistical analysis of GFP-positive cells with and without PPE2. (m) BS4 for detection of CRISPRa. (n) Detection of CRISPRa by BS4 through Arabidopsis protoplast transformation. (o) Statistical analysis of mCherry-positive cells with and without the CRISPR-Act3.0 activation system. Scale bar, $100 \mu \mathrm{m}$. All data are presented as the mean \pm SE. ( $n=5$ independent scopes). 
(a)

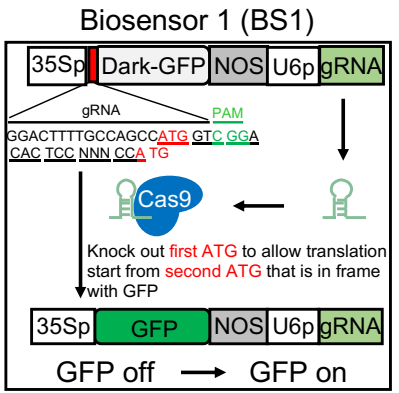

(c)

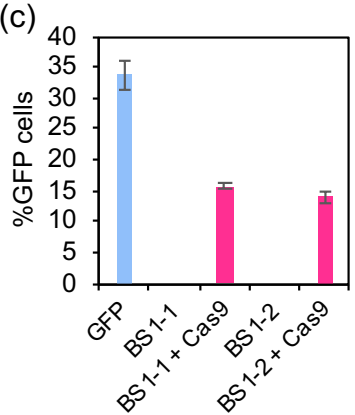

(f) $35 \mathrm{Sp}-\mathrm{GFP}$

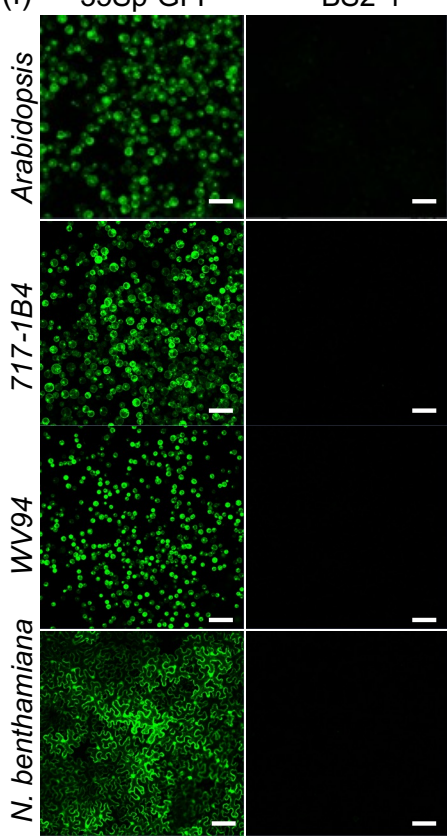

(d) Biosensor 2 (BS2)

\begin{tabular}{ll|l|l|}
\hline 35 Sp Dark-GFP & NOS U6p gRNA \\
\hline
\end{tabular}

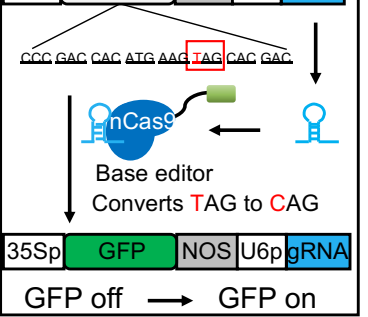

GFP off $\longrightarrow$ GFP on

BS2-1

BS2-1 + PABE-7

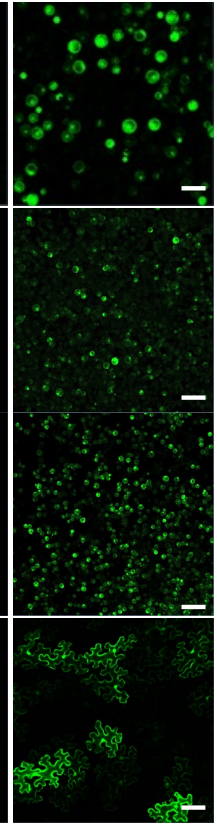

GFP

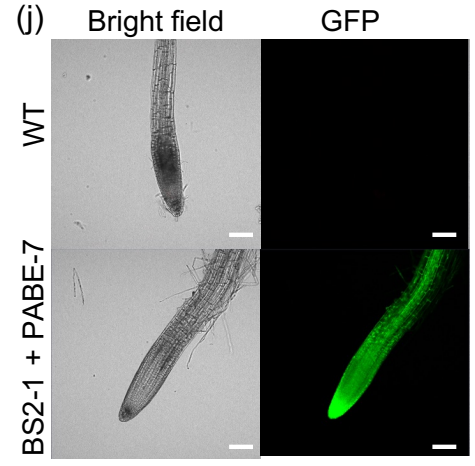

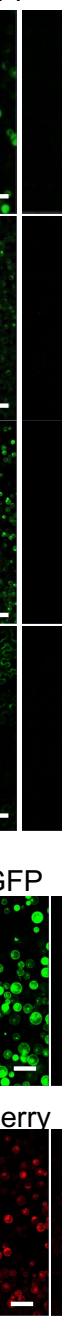

\section{BS2-2}

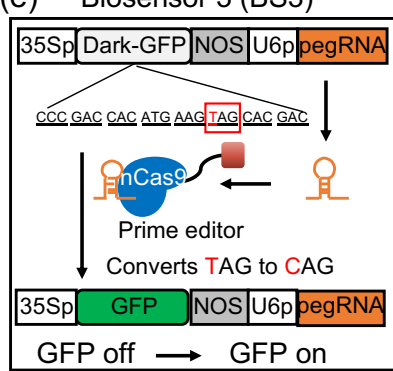

$\mathrm{BS} 2-2$ + PABE-7

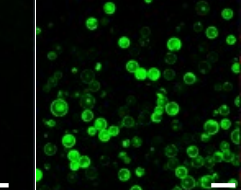

(k)

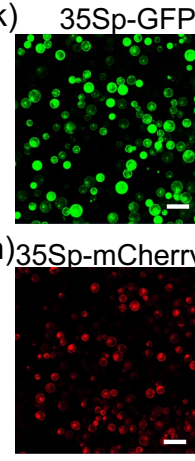

BS1-1

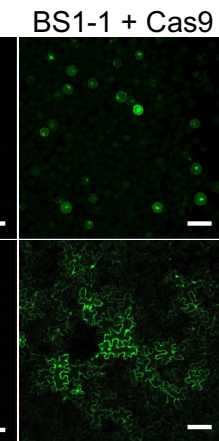

\section{$(9)$}

BS1-2 BS1-2 + Cas9

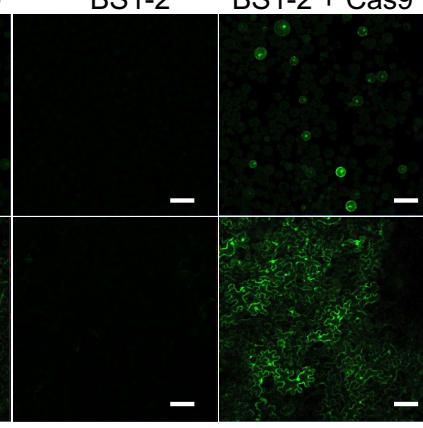

(g) 70

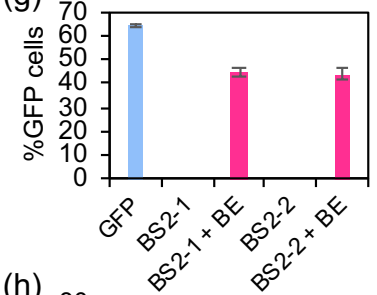

(h)

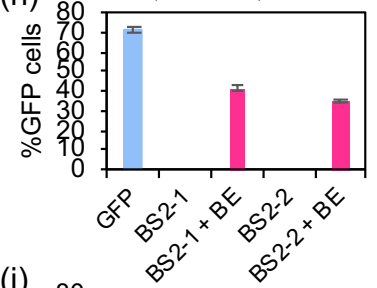

(i)

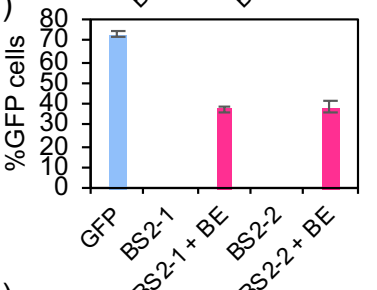

(I)

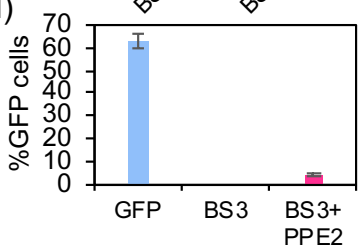

(m)

(o)
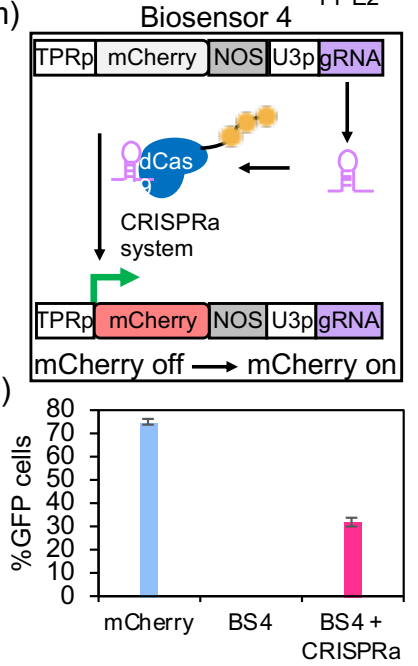